\title{
The conjunctiva in corneal epithelial wound healing
}

\author{
Harminder S Dua
}

\begin{abstract}
Backgroundlaims-During the healing of corneal epithelial wounds with limbal involvement, conjunctival epithelium often migrates across the denuded limbus to cover the corneal surface. It is believed that, over a period of time, conjunctival epithelium covering the cornea assumes characteristics of corneal epithelium by a process referred to as conjunctival transdifferentiation. The purpose of this study was to examine, clinically, the fate of conjunctival epithelial cells covering the cornea and to assess the healing of corneal epithelial wounds when the conjunctival epithelium was removed or actively prevented from crossing the limbus and extending onto the cornea.

Methods-10 patients with conjunctivalisation of the cornea were followed for an average of 7.5 months. Five patients in this group had their conjunctival epithelium removed from the corneal surface and allowed to heal from the remaining intact corneal epithelium. In another four patients with corneal epithelial defects, the conjunctival epithelium was actively prevented from crossing the limbus by mechanically scraping it off.
\end{abstract}

Results-The area of cornea covered by conjunctival epithelium appeared thin, irregular, attracted new vessels and was prone to recurrent erosions. Conjunctivalisation of the visual axis affected vision. Removal of conjunctival epithelium from the cornea allowed cells of corneal epithelial phenotype to cover the denuded area with alleviation of symptoms and improvement of vision. It was also established that migration of conjunctival epithelium onto corneal surface could be anticipated by close monitoring of the healing of corneal epithelial wounds, and prevented by scraping off conjunctival epithelium before it reached the limbus. Conclusion-This study shows that there is little clinical evidence to support the concept that conjunctival transdifferentiation per se, occurs in humans. "Replacement" of conjunctival epithelium by corneal epithelial cells may be an important mechanism by which conjunctival "transdifferentiation" may occur. In patients with partial stem cell deficiency this approach can be a useful and effective alternative to partial limbal transplantation, as is currently practised. (Br f Ophthalmol 1998;82:1407-1411)
The limbal epithelium, with its repository of stem cells, acts as a barrier that exerts an inhibitory growth pressure, preventing the migration of conjunctival epithelial cells onto the cornea. ${ }^{1}$ When a corneal epithelial defect involves the limbus, this barrier is lifted and conjunctival migration onto the cornea can occur. Apart from injury, several conditions affecting the ocular surface also result in stem cell deficiency and favour conjunctival epithelial migration on to the cornea. Important conditions include aniridia, Stevens-Johnson syndrome, burns caused by chemicals, heat or ionising radiation and chronic inflammation. ${ }^{2}$ Conjunctivalisation of the cornea can be partial or total. The corneal surface covered by conjunctival epithelium is characterised by the presence of goblet cells and is usually vascularised. ${ }^{3}$ It appears thin, irregular, and is prone to recurrent erosions. ${ }^{45}$ When it covers the pupillary area, vision can be significantly impaired. Conjunctival epithelium covering the cornea is believed to undergo a slow transformation to assume characteristics resembling corneal epithelium, a process referred to as conjunctival transdifferentiation. The management of stem cell deficiency includes keratoepithelioplasty and partial or total, allo- or autolimbal transplant. ${ }^{6}$

The purpose of this study was twofold: (1) to examine clinically whether conjunctival transdifferentiation occurs, over an extended period of time, in the area of cornea covered by conjunctival epithelium, and (2) in patients with partial stem cell deficiency, to examine whether mechanical debridement of conjunctival epithelium, before or after it had crossed the limbus to cover the cornea, would allow adequate limbal and corneal epithelial healing to occur from the remaining intact limbal (stem cell) epithelium.

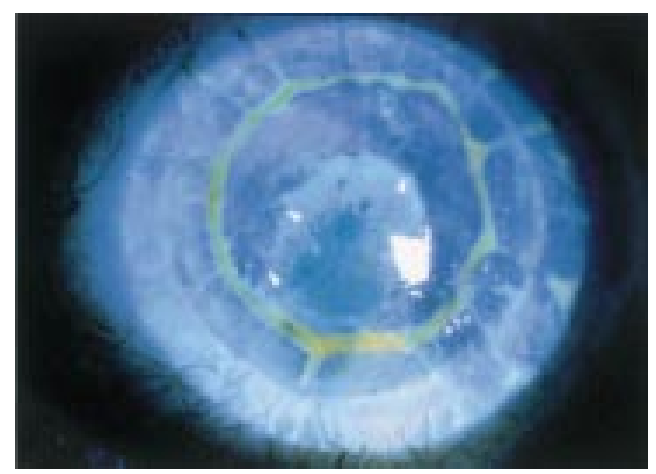

Figure 1 Slit lamp diffuse view of a fluorescein stained cornea of a patient with a corneal graft showing a clear demarcation between corneal and conjunctival epithelial phenotypes. The pupillary area is covered by conjunctival epithelium. Tiny "buds" of corneal epithelium can be seen along the line of contact between corneal and conjunctival epithelium (arrowheads) $(\times 10)$. 


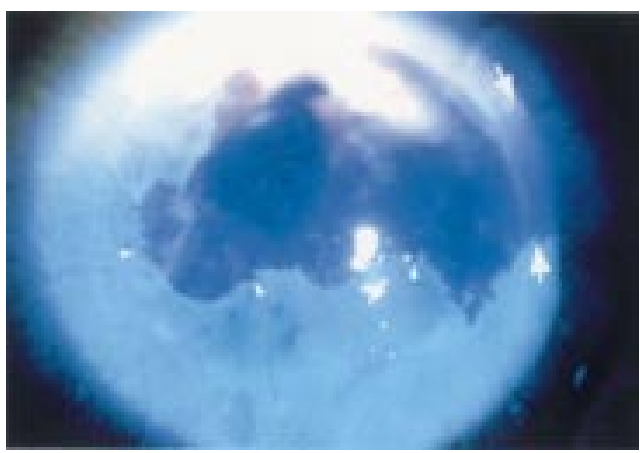

Figure 2 Slit lamp diffuse view of a fluorescein stained cornea of a patient with a corneal graft showing a clear demarcation between corneal and conjunctival epithelial phenotypes. The conjunctival epithelium shows light staining with fluorescein and blood vessels can be seen extending on the conjunctivalised epithelium. Tiny "buds" of corneal epithelium can be seen along the line of contact between corneal and conjunctival epithelium (arrowheads). The pupillary area is covered by corneal epithelium with is "sustained" by two clock hours of intact limbus (between large arrows). The best corrected visual acuity was 6/12. Scraping of conjunctival epithelium is not usually necessary in such cases $(\times 10)$.

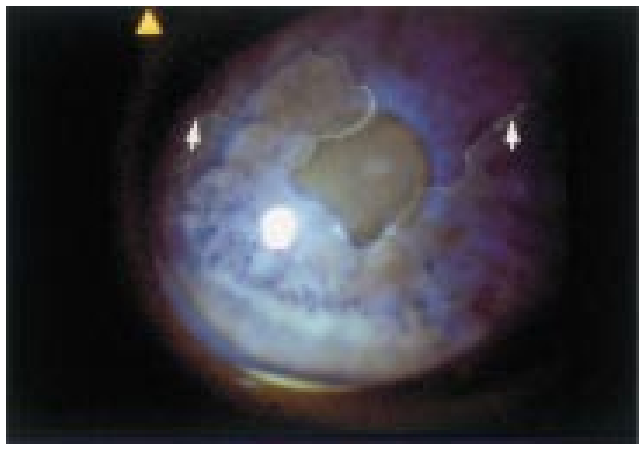

Figure 3 Slit lamp diffuse view of a fluorescein stained cornea of a patient with corneal surface problems following superficial chemical injury. The corneal surface shows a clear demarcation between corneal and conjunctival epithelial phenotypes. The conjunctival epithelium shows light staining with fluorescein. The pupillary area is covered by corneal epithelium with is "sustained" by four clock hours of intact limbus (between arrows). The best corrected visual acuity was 6/9. Scraping of conjunctival epithelium is not usually necessary in such cases $(\times 10)$.

\section{Patients and methods}

GROUP 1

Ten patients were included in the first part of the study. Four patients were observed pro- spectively during the healing of large ocular surface defects. A further six patients were recruited 2-5 years after complete ocular surface re-epithelialisation had occurred. Six patients had sustained chemical injury to the ocular surface, three were post-keratoplasty and the remaining one had conjunctivalisation of cornea following Stevens-Johnson syndrome as a child (Figs 1, 2, and 3). Patients were followed at 2-6 weekly intervals for 3-13 months with a mean of 7.5 months. All patients were examined by slit lamp biomicroscopy and fluorescein staining. Clinical photographs were taken at each visit and the area of corneal surface covered by conjunctival epithelium was estimated by planimetry as described before. Briefly, photographs of the cornea were photocopied onto graph paper, the area of the abrasion and the cornea was calculated, and the area of the corneal abrasion was expressed as a percentage of the total corneal area.

In five of these patients the conjunctival epithelium was mechanically scraped off the corneal surface (as described for group 2 below) and healing of the freshly denuded surface was allowed to proceed from the adjacent corneal epithelium. In two of these patients, only the pupillary area of the cornea, rather than the whole conjunctivalised surface of the cornea, was scraped. The patients were observed daily. Clinical details of patients are given in Table 1.

GROUP 2

In the second part of the study, four patients with ocular surface epithelial defects secondary to chemical injury were followed daily. The advancing sheet of conjunctival epithelium was prevented from crossing the limbus by mechanically scraping it off with a No 15 surgical blade under topical anaesthesia at the slit lamp. When the conjunctival epithelium reached to within a couple of millimetres of the limbus it was scraped back to approximately $5-7 \mathrm{~mm}$ away from the limbus. This procedure had to be repeated twice in three patients and was required only once in the fourth patient. Clinical details of these patients is given Table 2 .

Table 1 Clinical details of patients included in group 1 (conjunctival epithelium covering the cornea)

\begin{tabular}{|c|c|c|c|c|c|c|c|c|c|}
\hline \multirow[b]{2}{*}{ Age } & \multirow[b]{2}{*}{$\operatorname{Sex}$} & \multirow[b]{2}{*}{ Eye } & \multirow[b]{2}{*}{ Injury } & \multirow[b]{2}{*}{ Follow up } & \multicolumn{2}{|c|}{ Area of defect } & \multirow[b]{2}{*}{ Difference } & \multirow[b]{2}{*}{ Complications } & \multirow{2}{*}{$\begin{array}{l}\text { Corneal buds } \\
\text { at interface }\end{array}$} \\
\hline & & & & & Start & End & & & \\
\hline $45^{\star}$ & M & $\mathrm{R}$ & Detergent & 7 months & $58.6 \%$ & $56.1 \%$ & $2.5 \%$ & Fine vessels & Present \\
\hline $70^{\star}$ & M & $\mathrm{L}$ & Post PK & 12 months & $35 \%$ & $33.5 \%$ & $1.5 \%$ & $\begin{array}{l}\text { Vessels, filaments } \\
\text { Erosions } \times 2\end{array}$ & Present \\
\hline 71 & M & $\mathrm{L}$ & Post PK & 13 months & $26.4 \%$ & $26.5 \%$ & $-0.1 \%$ & Vessels & Present \\
\hline $54^{\star}$ & $\mathrm{F}$ & $\mathrm{L}$ & Detergent & 10 months & $28.8 \%$ & $23.2 \%$ & $5.6 \%$ & $\begin{array}{l}\text { Vessels, filaments } \\
\text { Erosions } \times 1\end{array}$ & Present \\
\hline 21 & M & $\mathrm{R}$ & Chemical & 5 months & $15 \%$ & $13 \%$ & $2 \%$ & $\begin{array}{l}\text { Fine vessels } \\
\text { Erosions } \times 1\end{array}$ & Present \\
\hline $40^{\star}$ & $\mathrm{F}$ & $\mathrm{R}$ & Detergent & 6 months & $86.2 \%$ & $85 \%$ & $1.2 \%$ & Vessels & Present \\
\hline 23 & M & $\mathrm{L}$ & Alcohol & 3 months & $18.6 \%$ & $15 \%$ & $3.6 \%$ & $\begin{array}{l}\text { Vessels, filaments } \\
\text { Erosions } \times 2\end{array}$ & Absent \\
\hline $38^{\star}$ & M & $\mathrm{R}$ & Alcohol & 8 months & $78.2 \%$ & $76 \%$ & $2.2 \%$ & $\begin{array}{l}\text { Vessels } \\
\text { Erosions } \times 1\end{array}$ & Present \\
\hline $32^{\star}$ & $\mathrm{F}$ & $\mathrm{L}$ & SJ syndrome & 3.5 months & $56.4 \%$ & $58.7 \%$ & $-2.3 \%$ & $\begin{array}{l}\text { Vessels, filaments } \\
\text { Erosions }>6\end{array}$ & \\
\hline 68 & M & $\mathrm{R}$ & Post PK & 4 months & $17.3 \%$ & $13 \%$ & $4.3 \%$ & Fine vessels & Absent \\
\hline
\end{tabular}

*Patients included in the study after complete re-epithelisation had occurred (follow up intervals for these patients are from the time they were included in the study).

tArea of defect refers to area covered by conjunctival epithelium. 
Table 2 Clinical details of patients included in group 2 (healing conjunctival epithelium prevented from extending across the limbus)

\begin{tabular}{|c|c|c|c|c|c|c|c|}
\hline Age & Sex & Eye & Injury & $\begin{array}{l}\text { Area of } \\
\text { defect }\end{array}$ & $\begin{array}{l}\text { Clock } \\
\text { hours of } \\
\text { limbus } \\
\text { involved }\end{array}$ & $\begin{array}{l}\text { No of } \\
\text { scrapes }\end{array}$ & $\begin{array}{l}\text { Duration } \\
\text { to complete } \\
\text { healing }\end{array}$ \\
\hline 47 & $M$ & $\mathrm{R}$ & Detergent & $58 \%$ & 4 & 2 & 14 days \\
\hline 22 & $M$ & $\mathrm{R}$ & Detergent & $42.4 \%$ & 4 & 2 & 12 days \\
\hline 28 & M & $\mathrm{R}$ & Alcohol & $60 \%$ & 5 & 2 & 14 days \\
\hline 31 & M & L & Detergent & $45 \%$ & 3 & 1 & 10 days \\
\hline
\end{tabular}

^Advancing sheet of healing conjunctiva was scraped 5-7 mm away from the limbus to allow limbal healing to complete.

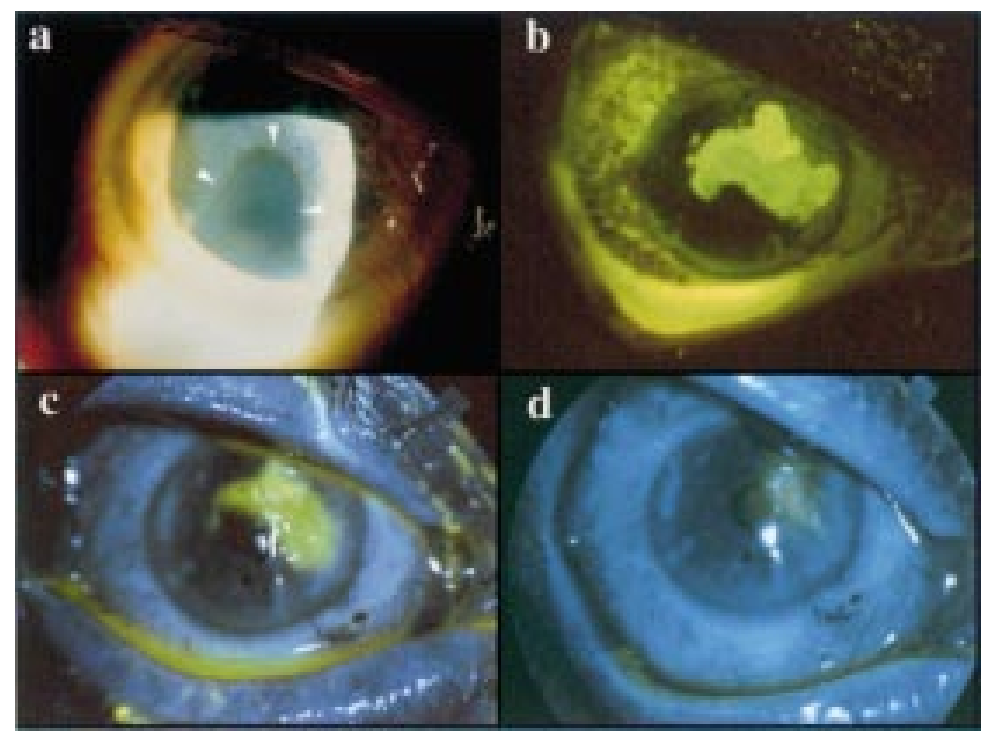

Figure 4 (a) Broad beam slit lamp photograph of a patient with extensive conjunctivalisation of the cornea. Only a small area of the corneal surface, corresponding to one and a half clock hours of the inferotemporal limbus, is covered by corneal epithelium. Note the well defined line of demarcation (arrowheads) between corneal and conjunctival epithelium. The patient's vision was $6 / 18(\times 10)$. (b) Fluorescein stained picture of the cornea immediately after removal of conjunctival epithelium over and around the pupillary area $(\times 6)$. (c) The same eye on day 2 following removal of conjunctival epithelium. Note that the pupillary area is being covered by migration of corneal epithelium from the small area of preserved corneal epithelium illustrated in $(a)(\times 6)$. (d) On day 5 following removal of conjunctival epithelium, healing is almost complete and the pupillary area is covered by healthy corneal epithelium. The visual acuity improved to $6 / 9(\times 6)$.

\section{Results (Tables 1 and 2)}

The area of cornea covered by conjunctival epithelium ranged from $15 \%$ to $86.2 \%$ at the outset. The area of "conjunctivalisation" had reduced by only $1.2 \%$ to $5.6 \%$ at the time of the last follow up visit. In one patient the area of conjunctivalisation had remained unchanged and in another it had increased by $2.3 \%$. In seven of the 10 patients, with a follow up of over 3 months, tiny buds of corneal epithelium could be seen protruding into the conjunctival epithelium all along the contact line between the two epithelial phenotypes (Figs 1 and 2). These buds were always seen arising from the corneal epithelium. Four patients had a total of seven episodes of recurrent erosions during the period of follow up. The one patient with conjunctivalisation, following StevensJohnson syndrome, alone had over six such episodes. Nine patients had a degree of superficial vascularisation which ranged from fine vessels extending just inside the limbus to well defined vessels extending $2-5 \mathrm{~mm}$ inside the limbus. Four patients had filamentary keratopathy limited to the area of "conjunctivalisation". In all patients the conjunctival epithelium covering the cornea was thinner relative

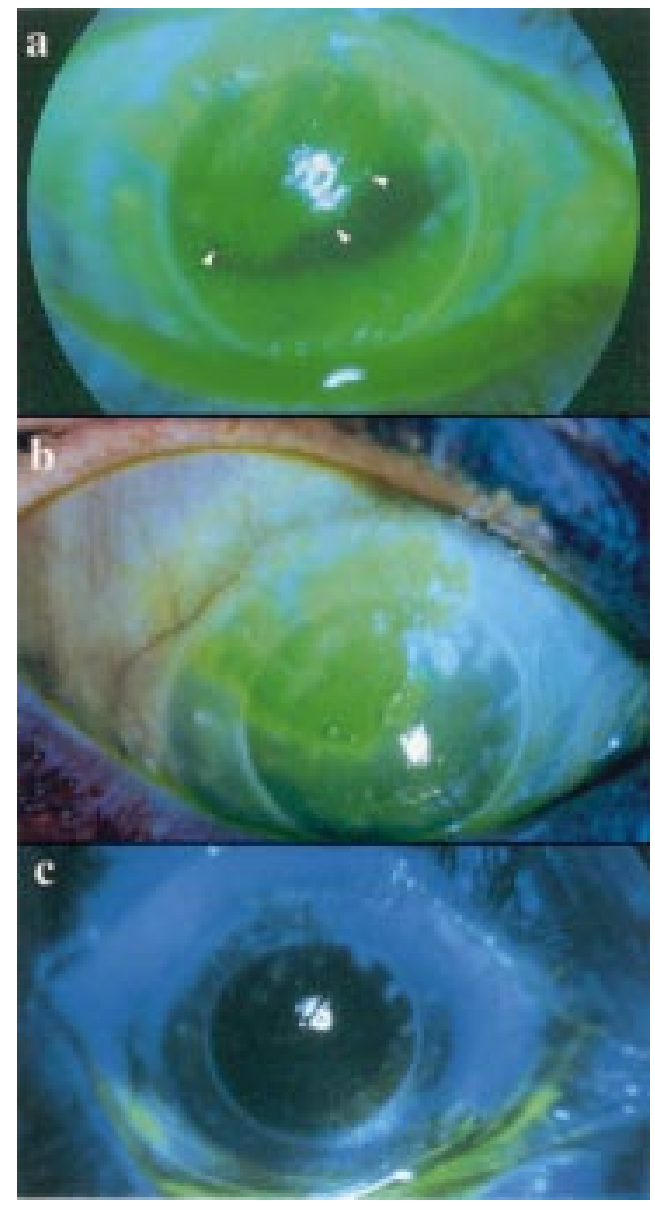

Figure 5 (a) Fluorescein stained photograph of a patient with a corneal graft showing just over $25 \%$ of the graft surface covered by conjunctival epithelium (arrowheads). The area appears irregular and shows light fluorescein staining. The patient was symptomatic and vision was a blurry $6 / 24(\times 10)$. (b) The same eye immediately following removal of the conjunctival epithelium $(\times 10)$. (c) After complete healing had occurred on day 5, the graft surface was covered by healthy corneal epithelium and the best corrected vision improved to 6/12 with alleviation of symptoms $(\times 6)$.

to the adjacent corneal epithelium. This was evidenced by pooling of fluorescein dye (Figs $1-3)$. In the five patients where conjunctival epithelium covering the corneal surface was mechanically removed, the entire denuded surface, or a large proportion of it, was covered by normal corneal epithelium within a week of debridement (Figs 4-7) with alleviation of symptoms and improvement of visual acuity (see legends of figures).

In all four patients in group 2 , the conjunctival epithelial sheet was successfully prevented from crossing the limbus until limbal healing was completed by the circumferentially migrating tongue-shaped sheets of limbal epithelium as described by Dua and Forrester. ${ }^{8}$

\section{Discussion}

That corneal defects could heal from the conjunctival epithelium has been known for a long time. ${ }^{9}$ Corneal epithelial wounds are known to stimulate a proliferative response in the perilimbal conjunctiva, ${ }^{10-12}$ but under normal circumstances, the limbal epithelium acts as a barrier and is able to exert an inhibitory growth 


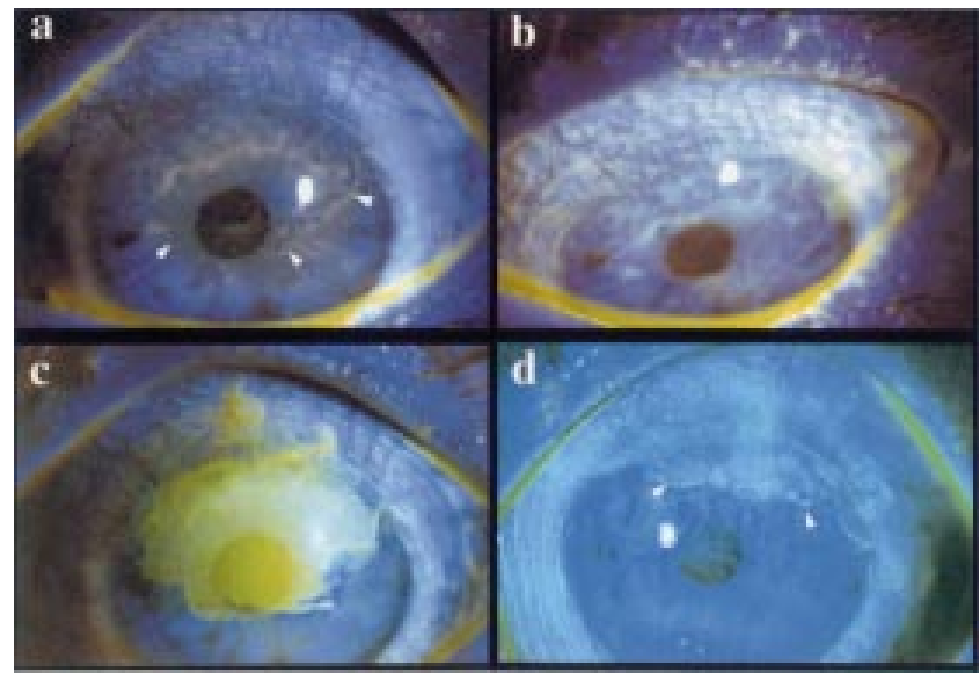

Figure 6 (a) Fluorescein stained photograph of a patient with Stevens-Fohnson syndrome who had over $50 \%$ of the superior corneal surface covered with conjunctival epithelium. The line of demarcation between corneal and conjunctival epithelium was not very well defined (arrowheads). The patient's vision was 6/12 and her main complaint was that of irritation and intermittent sharp pains due to recurrent erosions $(\times 10)$. (b) The same eye with the patient looking down to show that the superior conjunctiva too was showing coarse punctate staining with fluorescein and had injected vessels $(\times 10)$. (c) The eye after removal of the conjunctival epithelium from the pupillary area and superiorly. Note that the removal of epithelium was not extended across the limbus $(\times 10)$. (d) Six days following removal of conjunctival epithelium, the pupillary area and a part of the superior cornea is covered by healthy corneal epithelium. The demarcation between conjunctival epithelium and corneal epithelium is now well defined (arrowheads). The patient's vision improved to 6/5 but she was more impressed by the reduction in her symptoms $(\times 10)$.

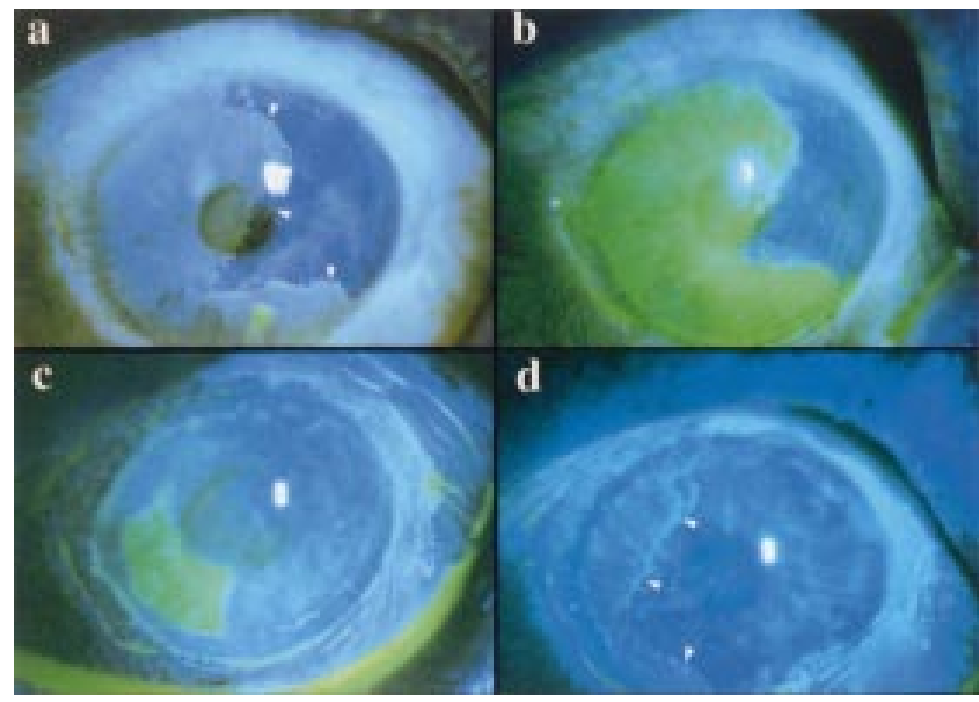

Figure 7 (a) Fluorescein stained photograph of the cornea of a patient who had presented several months after a chemical injury, showing a clear demarcation between corneal and conjunctival epithelial phenotypes (arrowheads). The pupillary area is almost entirely covered by conjunctival epithelium. Tiny "buds" of corneal epithelium can be seen along the line of contact between corneal and conjunctival epithelium. The patient's vision was 3/18 $(\times 10)$. (b) The eye after removal of all conjunctival epithelium from the corneal surface and limbus $(\times 10)$. (c) The eye on day 3 following removal of conjunctival epithelium. The corneal sheet has covered the pupillary area but the conjunctival epithelium has encroached on to cornea along the temporal limbus $(\times 10)$. (d) The eye after complete healing. A new line of contact is established between corneal and conjunctival epithelial phenotypes (arrowheads) but the pupillary area is covered by healthy corneal epithelium. The patient's vision improved to $6 / 9(\times 10)$.

pressure preventing migration of conjunctival epithelial cells onto the cornea. ${ }^{1}$ However, when the epithelial defect involves the limbus, this barrier is lifted and conjunctival migration onto the cornea occurs. This is associated with the appearance of goblet cells and, often, new blood vessels. ${ }^{3}$ It is believed that conjunctival epithelium covering the cornea undergoes a slow transformation to assume characteristics resembling corneal epithelium, a process referred to as conjunctival transdifferentiation. ${ }^{13}$ This process has been extensively studied in experimental animals where it has been shown that goblet cells do not migrate onto the cornea but develop de novo from non-goblet epithelial cells which can be identified by electron microscopy. Loss of goblet cells during transdifferentiation occurs by desquamation and in situ cell death. ${ }^{14}$ Vitamin $\mathrm{A}$ and vascularisation of regenerated epithelium are important factors that influence the process of transdifferentiation. ${ }^{15-18}$

The consensus from most animal studies is that although complete morphological transdifferentiation is possible biochemically and functionally it is far from satisfactory. ${ }^{19-21}$ Conjunctival transdifferentiation in animal models can therefore, at best, be described as squamous metaplasia with loss of goblet cells. Moreover, it has also been suggested that, in many of the above studies, conjunctival transdifferentiation could have occurred due to incomplete removal of limbal basal epithelium, ${ }^{22}$ with the result that regenerated epithelium demonstrated both corneal and conjunctival features without one actually changing to the other.

Dua and Forrester ${ }^{423}$ studied the healing, in humans, of large ocular surface epithelial wounds that involved the cornea, limbus, and conjunctiva. They identified two tongueshaped sheets of epithelium, arising from either end of the limbal defect, that showed a preferential circumferential migration along the limbus. In some patients they noted a centripetally migrating sheet of conjunctival epithelium that reached and migrated across the limbus, preventing the circumferentially migrating limbal sheets from meeting each other. As a result, varying areas of the cornea were covered by conjunctival epithelium. The epithelium in these areas was invariably thinner than adjoining normal corneal epithelium, showed a stippled stain with fluorescein, attracted new vessels, and was prone to recurrent erosions. This study confirms the above observations and demonstrates that a similar healing response also occurs in patients with corneal grafts. In all patients in this study, even several months on, the corneal surface covered by conjunctival cells remained relatively thin and irregular without clinically evident transdifferentiation. The difference in thickness sharply demarcated the area of "conjunctivalisation" from the adjacent healthy corneal epithelium and was rendered more obvious by the pooling of fluorescein dye. What was more interesting was that tiny buds of corneal epithelium could be seen protruding into the conjunctival epithelium all along the contact line between the two epithelial phenotypes. This observation has also been well illustrated in previous publications. ${ }^{48}$ These buds were always seen arising from the corneal epithelium and gave the impression that normal corneal epithelium was attempting to replace the conjunctival epithelium, gradually nudging it outward, towards the limbus. "Replacement" of conjunctival epithelium by normal corneal epithelium may 
therefore be yet another factor contributing to conjunctival "transdifferentiation". The results of this study would tend to support the view that complete conjunctival transdifferentiation probably does not occur in humans.

On the basis of these observations Dua et $a l^{423}$ had suggested that, although any kind of epithelial cover for the cornea may be a desirable goal in the short term, the ideal situation would be to encourage conjunctival epithelial cover for conjunctiva and corneal epithelial cover for cornea. They recommended that, in corneal epithelial defects with partial limbal involvement, conjunctival epithelium should be prevented from crossing the limbus until the circumferentially migrating sheets of limbal epithelium have met each other and the limbal barrier is re-established. This can be achieved by mechanically scraping the advancing conjunctival epithelial sheet and may have to be repeated two or three times because the conjunctival epithelium migrates rapidly compared to the limbal sheets. (While describing the procedure to the patients, the term "brushing" was adopted, to replace "mechanical scraping" as it sounds less invasive and was very acceptable to patients, causing much less anxiety and apprehension.) Such an approach would ensure corneal epithelial cover for the cornea and conjunctival epithelial cover for the conjunctiva. This hypothesis has been substantiated by the present study. On the other hand, in patients where conjunctival epithelium had already covered a part of the corneal surface at the time of presentation, it is easy to mechanically remove the conjunctival epithelium under topical anaesthesia at the slit lamp. This procedure was followed by rapid re-epithelisation of the cornea with corneal epithelium. Interestingly, it was the corneal epithelial sheet that rapidly advanced to cover the defect rather than conjunctival epithelium from the limbus. This observation, coupled with the presence of corneal epithelial buds described above, would suggest the presence of a constant and persistent drive in the corneal epithelial sheet to replace conjunctival epithelium. Furthermore, the observations made in this study indicate that although varying proportions of the corneal surface may be covered by conjunctival and corneal epithelium, the two phenotypes can achieve a state of equilibrium and "peacefully coexist". As long as the symptoms are tolerable and if the visual axis is covered by corneal epithelium, as seen in two patients in this study (Figs 2 and 3), removal of conjunctival epithelium is not necessary. In fact, if an extensive area of the cornea is covered by conjunctival epithelium, just sufficient conjunctival epithelium may be removed so as to allow the pupillary area of the cornea to be covered by corneal epithelium. This can lead to a significant improvement in vision. For this procedure to provide healthy corneal epithelial cover for denuded corneal surface it is important that some of the limbal (stem cell) epithelium was preserved at the time of the mechanical or chemical insult. It is not always necessary to undertake partial limbal or stem cell transplant to restore the corneal surface in such patients as was demonstrated in this study. If total corneal and limbal epithelium is lost and replaced by conjunctival epithelium, limbal (auto)transplants or keratoepithelioplasty should be considered.

The author wishes to express his gratitude to Paddy Tighe and April Powell Richards for their help in preparing the colour illustrations. HS Dua is the Julia Duane Scholar (Wills Eye Hospital, Philadelphia).

1 Tseng SCG. Concept and application of limbal stem cells. Eye 1989;3:141-57.

2 Coster DJ, Aggarwal RK, Williams KA. Surgical management of ocular surface disorders using conjunctival and stem cell allografts. Br F Ophthalmol 1995;79:977-82.

3 Thoft RA, Friend J, Murphy HS. Ocular surface epithelium and corneal vascularization in rabbits. I. The role of wounding. Invest Ophthalmol Vis Sci 1979;18:85-92.

4 Dua HS, Gomes JAP, Singh A. Corneal epithelial wound healing. Br f Ophthalmol 1994;78:401-8.

5 Dua HS. Stem cells of the ocular surface: scientific principles and clinical applications. Br f Ophthalmol 1995;

6 Holland EJ, Schwartz GS. The evolution of epithelial transplantation for severe ocular surface disease and a proposed classification system. Cornea 1996;15:549-56.

7 Dua HS, Forrester JV. Clinical patterns of corneal epithelial wound healing. Am f Ophthalmol 1987;104:481-9.

8 Dua HS, Forrester JV. The corneoscleral limbus in human corneal epithelial wound healing. Am f Ophthalmol 1990;110:646-56

9 Friedenwald JS. Growth pressure and metaplasia of conjunctival and corneal epithelium. Doc Ophthalmol 1951; 6:184-92.

10 Danjo S, Friend J, Thoft RA. Conjunctival epithelium in healing of corneal epithelial wounds. Invest Ophthalmol Vis Sci 1987;28:1445-9.

11 Haaskjold E, Bjerknes R, Refsum SB. Cell kinetics during healing of corneal epithelial wounds. Acta Ophthalmol (Copenh) 1989;67:174-80.

12 Haaskjold E, Sandvig KU, Bjerknes R, et al. The early cell kinetic response during healing of corneal epithelial wounds. Ophthalmic Surg 1992;23:680-4.

13 Shapiro MS, Friend J, Thoft RA. Corneal reepithelialization from the conjunctiva. Invest Ophthalmol Vis Sci 1981;21:135-42.

14 Aitken D, Friend J, Thoft RA. Corneal re-epithelialization from the conjunctiva. Invest Ophthalmol Vis Sci 1988;29: 224-31.

15 Tseng SCG, Hirst LW, Farazdaghi M, et al. Goblet cell density and vascularization during conjunctival transdifferentiation. Invest Ophthalmol Vis Sci 1984;25:1168-76.

16 Huang AJW, Watson BD, Hernandez E, et al. Induction of conjunctival transdifferentiation on vascularized corneas by photothrombotic occlusion of corneal vascularization. Ophthalmology 1988;95:228-35.

17 Tseng SCG, Hirst LW, Farazdaghi $M$, et al. Inhibition of conjunctival transdifferentiation by topical retinoids. Invest Ophthalmol Vis Sci 1987;28:538-42.

18 Tseng SCG, Farazdaghi M, Rider AA. Conjunctival transdifferentiation induced by systemic vitamin A deficiency in vascularized rabbit corneas. Invest Ophthalmol Vis Sci 1987;28:1497-504

19 Thoft RA, Friend J. Biochemical transformation of regenerating ocular surface epithelium. Invest Ophthalmol 1977;16: $14-20$.

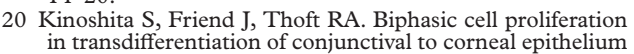
in rabbits. Invest Ophthalmol Vis Sci 1983;24:1008-14.

21 Harris TM, Berry ER, Pakurar AS, et al. Biochemical transformation of bulbar conjunctiva into corneal epithelium: an electrophoretic analysis. Exp Eye Res 1985;41:597-605.

22 Kruse FE, Chen JJY, Tsai RJF, et al. Conjunctival transdifferentiation is due to incomplete removal of limbal basal epithelium. Invest Ophthalmol Vis Sci 1990;31:1903-13.

23 Dua HS, Forrester JV, Cohen EJ, et al. Clinical observations on corneal epithelial cell migration in humans. Invest Ophthalmol Vis Sci 1993;34(suppl):1017. 